\title{
Morphological Observation of Specific Condensation Effect of Cholesterol on Dipalmitoyl Phosphatidyl Choline (DPPC) Monolayer by Dropping Method
}

\section{Takashi Yokoyama1, Daisuke Yoshida1, Hiroya Mori'1, Masaya Okabe1, Zameer Shervani' Keijiro Taga1, Yasushi Yamamoto', Ayumi Sumino3 ${ }^{3}$, Takehisa Dewa3, Mamoru Nango ${ }^{3}$, Masato Yamamoto ${ }^{4}$}

\author{
${ }^{1}$ Department of Materials Science and Engineering, Nagoya Institute of Technology, Nagoya, Japan \\ ${ }^{2}$ Tohoku Foods Company, Sendai, Japan \\ ${ }^{3}$ Department of Frontier Materials, Nagoya Institute of Technology, Nagoya, Japan \\ ${ }^{4}$ Department of Chemistry, School of Arts and Sciences, Showa University, Fujiyoshida, Japan \\ Email: shervani.nanotec@gmail.com
}

How to cite this paper: Yokoyama, T., Yoshida, D., Mori, H., Okabe, M., Shervani, Z., Taga, K., Yamamoto, Y., Sumino, A., Dewa, T., Nango, M. and Yamamoto, M. (2016) Morphological Observation of Specific Condensation Effect of Cholesterol on Dipalmitoyl Phosphatidyl Choline (DPPC) Monolayer by Dropping Method. Journal of Biophysical Chemistry, 7, 98-109. http://dx.doi.org/10.4236/jbpc.2016.74009

Received: May 8, 2016

Accepted: October 9, 2016

Published: October 14, 2016

Copyright $\odot 2016$ by authors and Scientific Research Publishing Inc. This work is licensed under the Creative Commons Attribution International License (CC BY 4.0).

http://creativecommons.org/licenses/by/4.0/

\begin{abstract}
Morphology of dipalmitoyl phosphatidyl choline (DPPC)-cholesterol (Chol) mixed monolayer formed on water surface by dropping method was investigated using surface tension measurement (STm), Brewster angle microscopy (BAM), and fluorescence microscopy (FM). STm showed strong condensation effect of Chol in fluidic DPPC monolayer. Excess area $\left(\mathrm{S}_{\mathrm{ex}}\right)$ from mean mixing state of DPPC and Chol was about twice larger than that by general compression method in the range from $x_{C}=$ 0.2 to 0.4 ( $x_{C}$ : mole fraction of Chol). BAM and FM images showed clearly that the fluidic DPPC monolayer changed to condensed rigid monolayer due to the condensation effect of Chol. At more than $x_{C}=0.3$ DPPC-Chol mixed monolayer changed to condensed state similar to the Chol monolayer. These results support previous reports by compression method that Chol molecule demonstrates the strong condensation effect to the fluidic monolayer and also indicate that dropping method enables to form unique monolayer on the water surface.
\end{abstract}

\section{Keywords}

DPPC-Chol Mixed Monolayer, Dropping Method, Surface Tension Measurement, Brewster Angle Microscopy, Fluorescence Microscopy, Condensation Effect 


\section{Introduction}

Biomembranes have fundamental fluid-structures similar to "fluid mosaic model" proposed by Singer and Nicolson [1]. Biomembranes are composed of various kinds of phospholipids in which functional proteins are buried [2]. Membrane-fluidity is important for functioning of membrane such as molecular recognition, transport of nutrition and various other solutes, and construction of tertiary structures of protein [3] [4].

Membrane lipids contain 30wt\% cholesterol (Chol) and Chol is an important membrane component which controls the membrane fluidity [5] [6]. Chol gives membrane-peculiar flexibility and keeps membrane structure balance in range of large temperature [2]-[4]. Apart from membrane flexibility, Chol is responsible for various functional activities of membrane protein and cell. Therefore, Chol is one of essential component in biological phenomenon.

Lipid monolayer formed on water surface presents half the membrane and has been used as model-membrane because easy preparation and investigation [7]. Recently, we have prepared and studied monolayer formation by dropping method [8]. In dropping method, solution droplets are dropped continuously on water surface at regular time interval. We have reported using various physicochemical methods that dipalmitoyl phosphatidyl choline (DPPC) monolayer formed by dropping method maintained its fluidity and flexibility and the limiting molecular area was larger than measured by general compression method. Monolayer by dropping method would be likely to apply to various property investigations as bio-mimetic membrane [9] [10].

In this communication, we have investigated Chol mixing-effect on fluidic DPPC monolayer formed by dropping method for various Chol mole fractions using surface tension measurement (STm), Brewster angle microscopy (BAM), and fluorescence microscopy (FM). We have also discussed the "condensation effect" of Chol where Chol attracted monolayer molecules of DPPC and made monolayer rigid.

\section{Experimental}

\subsection{Materials}

Dipalmitoyl phosphatidyl choline (DPPC, purity: $>99 \%$ ) and Cholesterol (Chol, purity: $>99 \%$ ) were purchased from Avanti polar lipids Inc. (Alabama, USA) and SigmaAldrich Corp. (St. Louis, USA), respectively and were used without further purification. Purified water with conductance of less than $0.06 \mu \mathrm{S} / \mathrm{cm}$ was obtained using a Super Water Purifying System (WL-21P; Yamato Scientific Corp. Ltd., Tokyo, Japan).

\subsection{Monolayer Formation}

DPPC and Chol of respective amount were dissolved in chloroform (purity: 99\%, Wako Pure Chem. Ind. Ltd., Osaka, Japan) to prepare lipid solutions for monolayer preparation. Solution of lipids in chloroform was spread on a purified water surface with a 100 $\mu \mathrm{l}$ microsyringe (Ge-0583-04; Hamilton Corp., Nevada, USA) to prepare monolayer. The details of the method of monolayer formation have been reported in earlier publi- 
cation [8]. $1 \mu \mathrm{l}$ drop of lipids in chloroform solution was dropped gently on the water surface and next was dropped after $>1$ min so that previous droplet expanded on the water surface. The expanding process was also confirmed that the value of surface tension (Section 2-3) became constant after each dropping. This action was repeated until mixed monolayer was formed, and the formation was confirmed that the formation of lenses was observed on the water surface and the value of surface tension (Section 2-3) did not change.

\subsection{Surface Tension Measurement (STm)}

STm for mixed monolayers was carried out on a Surface Tensiometer (CBVP-A3; Kyowa Interface Science Corp. Ltd., Saitama, Japan) with a platinum Welhelmy plate. Surface pressure (SP) of each monolayer was recorded as a function of the dropping volume (molecule numbers) at $26^{\circ} \mathrm{C} \pm 1^{\circ} \mathrm{C}$.

\subsection{Brewster Angle Microscopy (BAM)}

BAM for visualizing the morphologies of each mixed monolayer was carried out on a BAM microscope (EMM633K; Filgen Inc., Nagoya, Japan) attached with a USB-CAP type (SD-USB2CUP3; AREA Co. Ltd., Tokyo, Japan) imaging analysis software. The lateral resolution of BAM was about $1 \mu \mathrm{m}$. P-polarized light of $632.8 \mathrm{~nm}$ wavelength from a $10 \mathrm{~mW}$ He-Ne laser was irradiated at the Brewster angle of $53.1^{\circ}$ on each mixed monolayer surface. The reflected light was magnified with a $40 \mathrm{~mm}$ focal length lens and detected by a CCD camera (C5948-70; Hamamatsu Photonics, Hamamatsu, Japan). The formation process of each mixed monolayer was observed on real-time at $26^{\circ} \mathrm{C} \pm$ $1^{\circ} \mathrm{C}$.

\subsection{Fluorescence Microscopy (FM)}

FM for visualizing the morphologies of each mixed monolayer was carried out on a total internal reflection fluorescence (TIRF) microscope (TE2000-U; Nikon Co., Tokyo, Japan) at room temperature. FM microscope was equipped with an oil immersion objective lens (Plan Apo $100 \times \mathrm{H}$, numerical aperture = 1.45; Nikon Co., Tokyo, Japan), a $575 \mathrm{~nm}$ dichroic mirror, a set of barrier filters (590 nm long pass and $765-855 \mathrm{~nm}$ band pass filters), a laser with $\lambda=532 \mathrm{~nm}$ (CL532-025-L, $25 \mathrm{~mW}$; Crystallizer, Reno, USA), a cooled CCD camera (ORCA-ER; Hamamatsu Photonics, Hamamatsu, Japan), and AQUA-COSMOS imaging analysis software. The lateral resolution of FM was about $0.1 \mu \mathrm{m}$.

The details of sample preparation for FM have been reported in earlier publication [8]. Fluorescent probe of rhodamine-dimiyrstoyl phosphoethanolamine (Rh-DMPE, Avanti Polar Lipids Inc., Alabama, USA) was incorporated at $0.1 \mathrm{~mol} \%$ in the mixtures-chloroform solution. After a monolayer was formed on the water surface at $26^{\circ} \mathrm{C}$ $\pm 1^{\circ} \mathrm{C}$, a cover glass sunk previously in the water phase was scooped horizontally from under the monolayer using scooping-up method [11]. The sample preparation of each monolayer for FM observation was done separately. 


\section{Results and Discussion}

\subsection{Surface Tension Measurement (STM)}

Figure 1 shows surface pressure (SP) versus molecular area $(\pi-A)$ isotherm of various DPPC-Chol mixed monolayers (mole fraction of Chol: 0.0, 0.1, 0.2, 0.3, 0.4, 0.6, 0.8, $0.1)$ at $26^{\circ} \mathrm{C}$ using dropping method. The horizontal axis represents molecular area calculated from molecular numbers in the dropping volume. The vertical axis represents SP.

On the pure DPPC monolayer, the SP increases gradually from a large molecular area $\left(>1.5 \mathrm{~nm}^{2}\right)$ and it was Liquid-Expand (LE) phase. A small transition noticed around $12 \mathrm{mN} / \mathrm{m}$, after SP increased up to $45 \mathrm{mN} / \mathrm{m}$. The limiting molecular area $\left(A_{0}\right)$ of DPPC monolayer was $0.62 \pm 0.05 \mathrm{~nm}^{2} /$ molecule $(40 \mathrm{mN} / \mathrm{m})$. This curve is almost the same as that we reported previously [8] and also different from that by compression method [12]. In compression method, the maximum SP was $50 \mathrm{mN} / \mathrm{m}$ and $A_{0}$ was 0.48 $\mathrm{nm}^{2} /$ molecule. On the other hand, our maximum SP was $10 \%$ lower $(45 \mathrm{mN} / \mathrm{m})$ than that by compression method and $A_{0}$ was also ca. $30 \%$ larger $\left(0.62 \mathrm{~nm}^{2} /\right.$ molecule $)$ than that by compression method. Therefore a "semi-expanded state" type DPPC monolayer was formed on water surface and it has a "fluid structure" that the hydrophilic group of DPPC molecules (phosphatidyl choline group) and water molecules structure themselves in the most comfortable hydrogen-bonding network (hydrophilic interaction). We defined this state as "semi Liquid-Condensed (semi-LC) phase" reported previously [8].

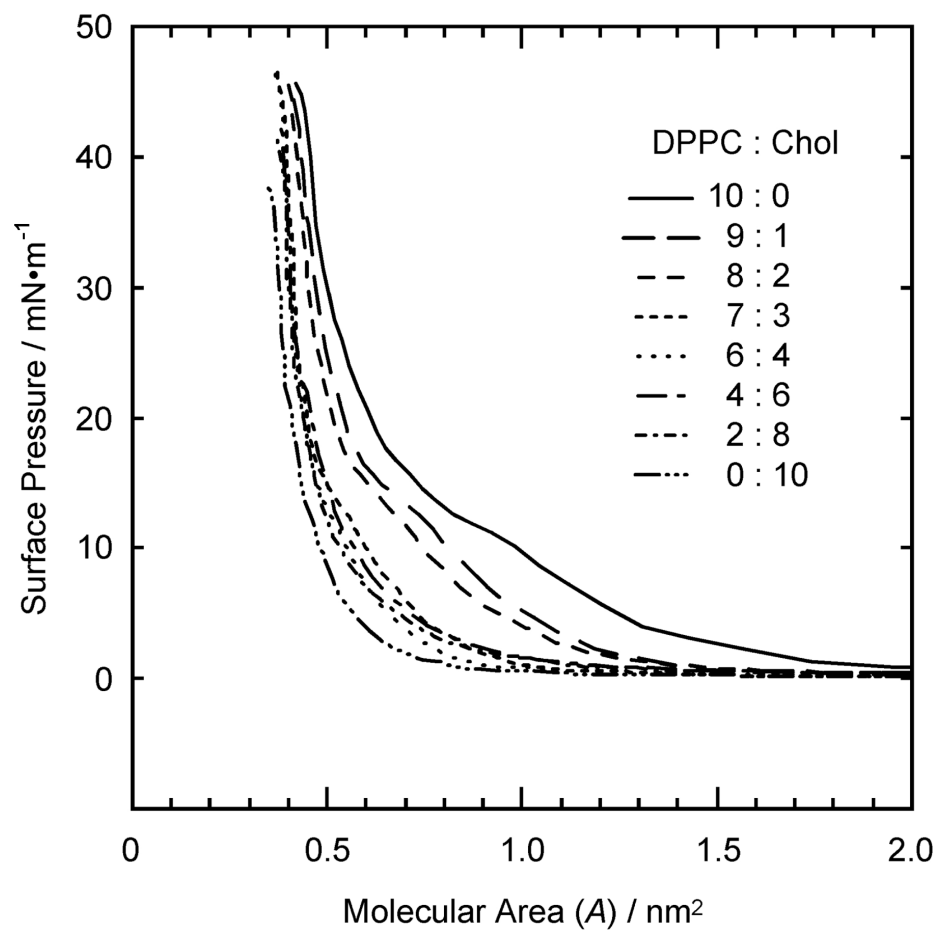

Figure 1. Surface pressure versus molecular area $(\pi-A)$ isotherm curves of DPPC-Chol mixed monolayers on the air/water interface at $26^{\circ} \mathrm{C}$. 
According to an increase in the mole fraction of Chol, the curves of DPPC-Chol mixed monolayer shifted to the left and approached to the pure Chol monolayer. The transition that revealed in the pure DPPC monolayer was not clear more than 0.3 of Chol mole fraction and the shape of curves became closely similar to that of pure Chol monolayer. This indicates that at more than 0.3 of Chol mole fraction condensed mixed monolayers are formed on the water surface. Therefore, mixed monolayer at less than 0.3 forms semi-LC phase and more than 0.3 condensed phase.

On the pure Chol monolayer, the curve showed a gradual increase in SP from more than $0.8 \mathrm{~nm}^{2}$ of molecular area and the SP increased up to $37 \mathrm{mN} / \mathrm{m}$. $A_{0}$ was $0.41 \pm 0.02$ $\mathrm{nm}^{2} /$ molecule at $34 \mathrm{mN} / \mathrm{m}$. This value was similar to that by compression method reported previously [12] [13]. On the other hand, the shape of curve was a little different from previous ones. Dropping in succession by dropping method occurs the disentanglement of unbalanced domain of Chol molecules because of the redissolution of domain. This supplies the dispersion of Chol molecules all over the water surface and thereby the increase in the SP from the initial stage. Such a peculiar characteristic by dropping method may appear in our Chol monolayer curve. A peculiar characteristic by dropping method will be described in cholesterol monolayer curve.

The mean limiting molecular area of a DPPC-Chol mixed monolayer in this study can be compared to that of the ideal mixed monolayer which is calculated from each $A_{0}$ of pure DPPC and Chol monolayer to obtain the excess area $S_{e x}$ [14] [15] by using the following equation:

$$
S_{e x}=S_{D C}-\left(x_{D} S_{D}+x_{C} S_{C}\right)
$$

where $S_{D C}$ is the mean limiting molecular area of the mixed monolayer, $S_{D}$ and $S_{C}$ the limiting molecular areas of pure DPPC and Chol, respectively. $x_{D}$ and $x_{C}$ are the mole fractions of the DPPC and Chol components. Figure 2 shows Chol mole fraction dependence of $S_{e x}$ We also showed typical Chol mole fraction dependences of $S_{e x}$ of previously reported data obtained by compression method, which those data were re-calculated by our calculation method, based on the reported $\pi$ - $A$ isotherm curves [12] [13]. The shape of $S_{e x} v s$. Chol mole fraction plot was similar to that of previous data. Therefore, the addition of Chol in the DPPC monolayer showed the negative deviation. This result indicates again that Chol molecules have the condensation effect to DPPC monolayer [16] [17]. $S_{e x}$ value obtained in our experiment was about twice larger than that of previously reported data in $x_{C}$ range from 0.2 to 0.4 , despite that our $S_{e x}$ of $x_{C}=$ 0.1 and more than 0.5 coincided almost with the previous data.

As mentioned above, our DPPC monolayer formed by dropping method has fluidity-rich structure where hydrophilic interaction between phosphatidyl choline groups and water molecules are more than hydrophobic alkyl chains interaction. This state is different from that by compression method where the hydrophobic interactions are dominant to the formation of the monolayer because of the action of artificial force. The condensation effect of Chol on more fluidic DPPC monolayer, prepared in our laboratory [8], is stronger than previous data. Hydrophobic Chol molecules strongly attract weakly interacted alkyl chains in DPPC molecules. In other words, the existence of 


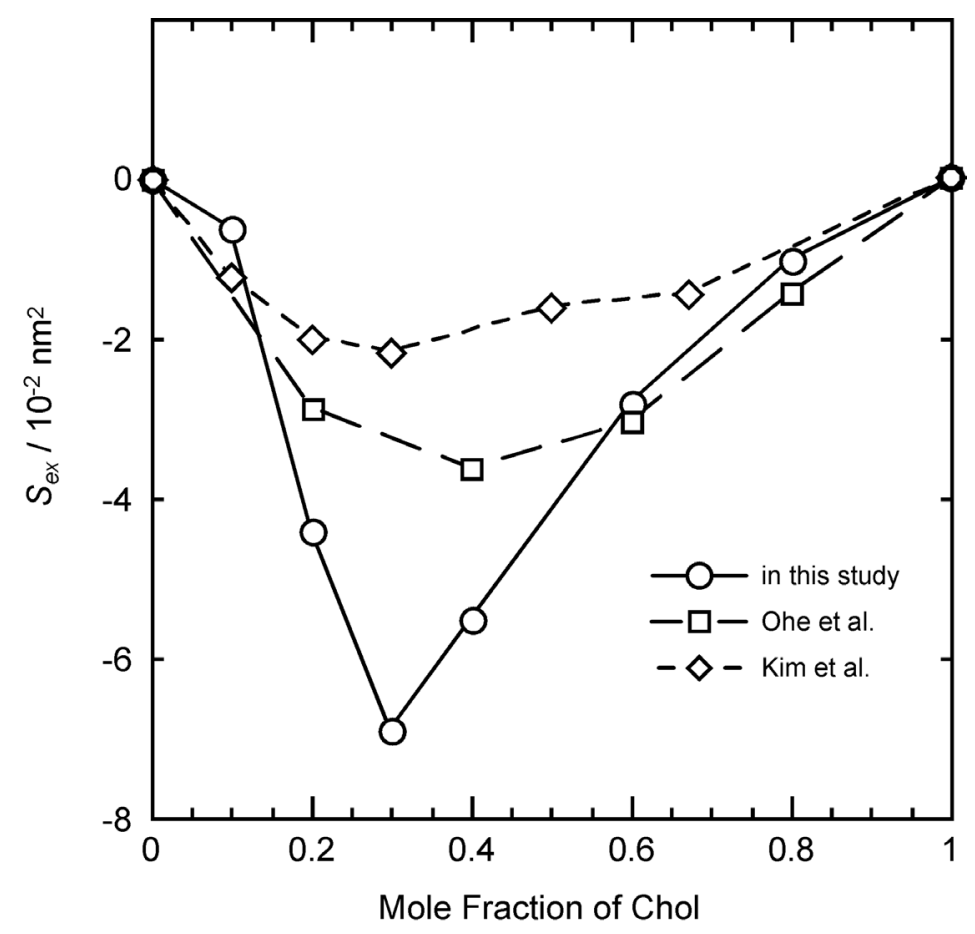

Figure 2. Excess mean limiting molecular area $S_{e x}$ of DPPC-Chol mixed monolayers on the air/water interface, including typical data of Ohe et al. [12] and Kim et al. [13].

large $S_{e x}$ by the addition of Chol indicates that fluidity-rich pure DPPC monolayer was formed on the water surface by dropping method.

\subsection{Brewster Angle Microscopy (BAM)}

Figure 3 shows a series of typical BAM images on DPPC-Chol mixed monolayers with four mole fractions of Chol $\left(x_{C}=0.0,0.2,0.3,1.0\right)$. (a)-(d) were pure DPPC monolayer $\left(x_{C}=0.0\right)$. (e)-(h) and (i)-(l) were each DPPC-Chol mixed monolayer of $x_{C}=0.2$ and 0.3 , respectively. $(\mathrm{m})-(\mathrm{p})$ were pure Chol monolayer $\left(x_{C}=1.0\right)$. BAM observation was performed on each monolayer at the dropping volume corresponding to four SPs of the monolayer.

Images of pure DPPC monolayer ((a)-(d) in Figure 3) resembled with previously reported experiments [8]. We briefly describe the essential points below. Before the SP of a transition ( $\leq 8 \mathrm{mN} / \mathrm{m}$, (a) and (b)), BAM image gradually became bright with increase in SP (dropping volume); the monolayer was beginning to form on the water surface. At the transition of $12 \mathrm{mN} / \mathrm{m}$ (c), the image showed the coexistence state that has hole-type dark parts in whole bright domain (spot-type pattern). This state was contrary to that by compression method and indicates that the hydrophilic monolayer which is easy to match water molecules is formed over the whole water surface. At high SP $(42 \mathrm{mN} / \mathrm{m},(\mathrm{d}))$, the image showed homogeneous bright state. This unique formation process different to that by compression method indicates that fluidic DPPC monolayer was formed by dropping method. The result corresponded to that observed by STm (Figure 1). 

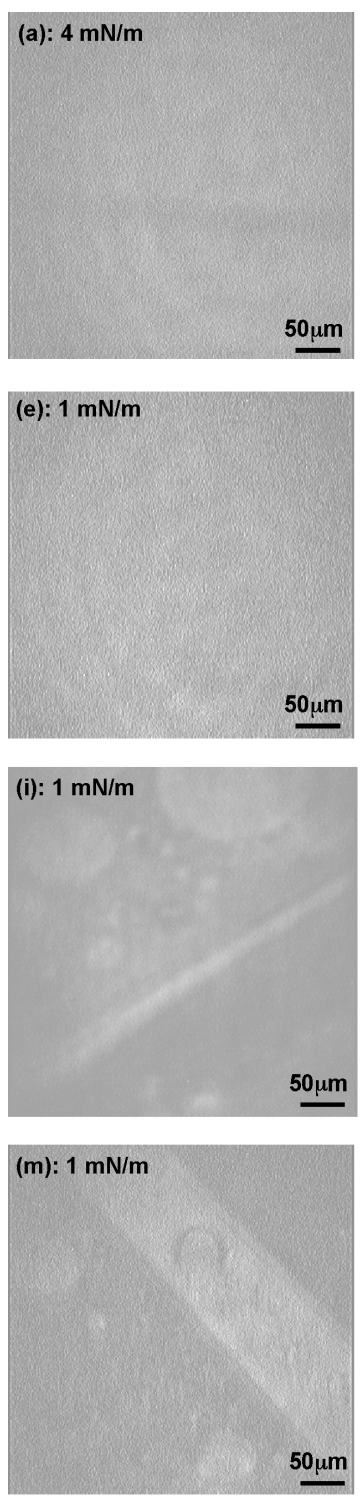
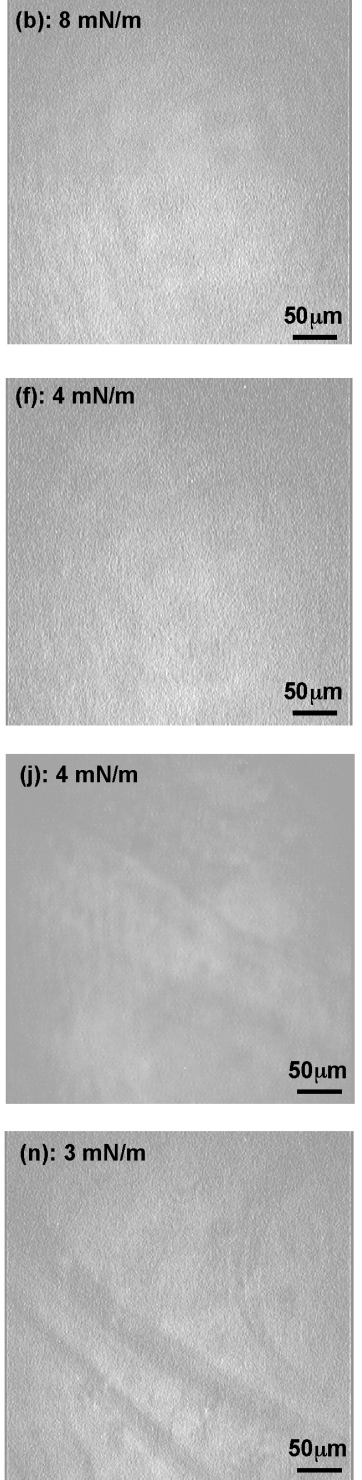
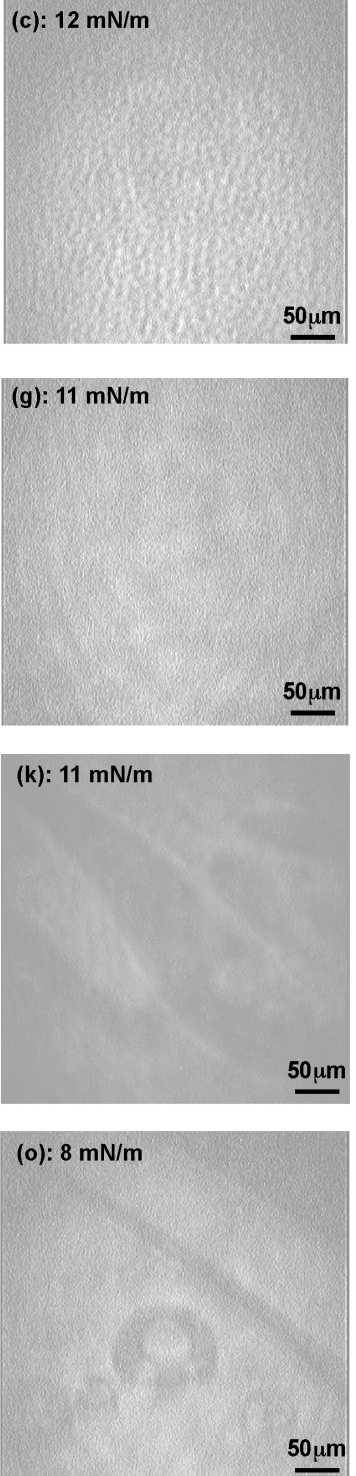
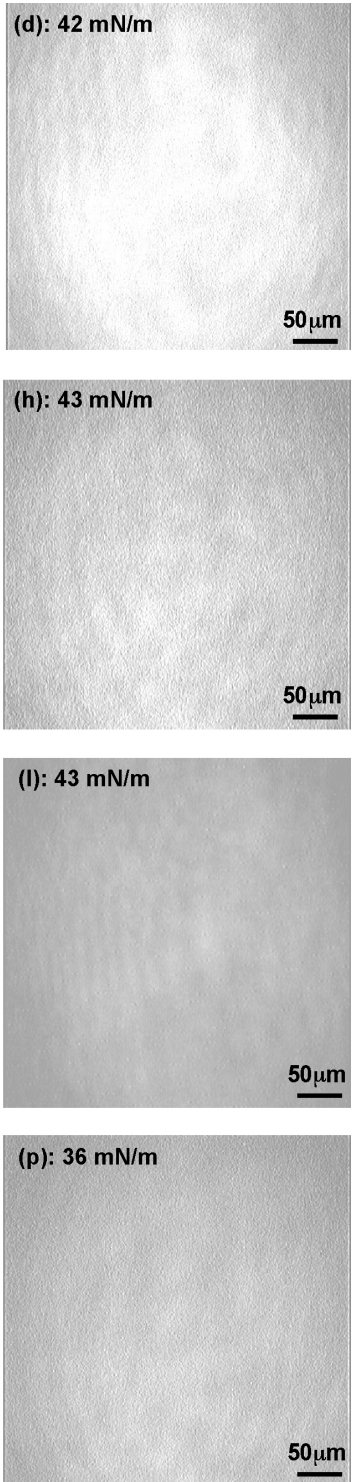

Figure 3. BAM images of DPPC-Chol mixed monolayer at various surface pressures. (a)-(d): Pure DPPC monolayer [8], (e)-(h): Mixed monolayer of $x_{C}=0.2$, (i)-(l): Mixed monolayer of $x_{C}=0.3,(\mathrm{~m})$-(p): pure Chol monolayer.

On DPPC-Chol mixed monolayer of $x_{C}=0.2((\mathrm{e})$-(h) in Figure 3), the condensation effect of Chol remarkably revealed in the BAM image. At quite a low SP of $1 \mathrm{mN} / \mathrm{m}$, the image showed bright state ((e)) compared to that of pure DPPC monolayer at $4 \mathrm{mN} / \mathrm{m}$ ((a)). This indicates that Chol molecules assemble DPPC molecules at $1 \mathrm{mN} / \mathrm{m}$. At 4 $\mathrm{mN} / \mathrm{m}$, the image showed spot-type pattern ((f): Indistinct image because of the low resolution of BAM) and the size of dark spot was a little smaller than that of pure DPPC monolayer ((c)). We could also obtain a clear spot image at FM observation (Figure 4(f): Section 3-3). On the pure DPPC monolayer, the appearance of spot-type pattern ((c)) corresponded to the transition of the monolayer (Figure 1). Therefore, it was found that the transition of mixed monolayer of $x_{C}=0.2$ occurred at $4 \mathrm{mN} / \mathrm{m}$. This value was $8 \mathrm{mN} / \mathrm{m}$ lower than that of pure DPPC monolayer $(12 \mathrm{mN} / \mathrm{m})$. Chol mole- 

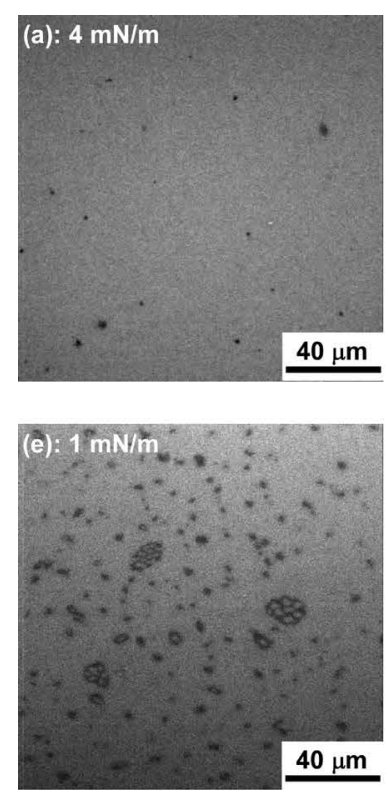
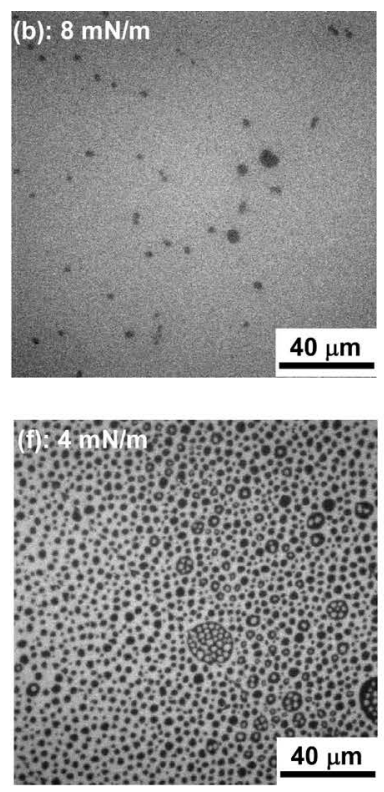
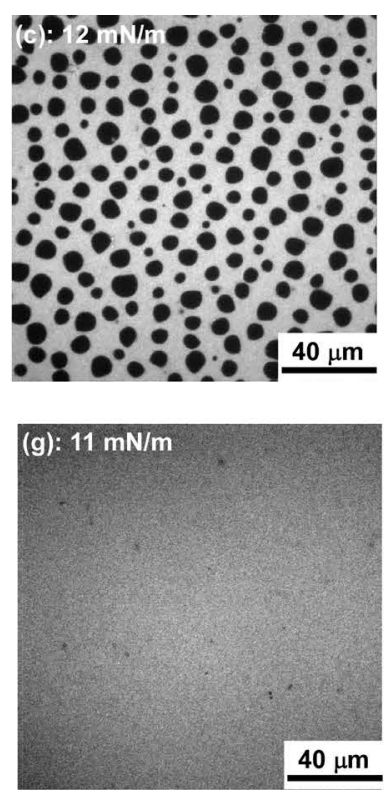
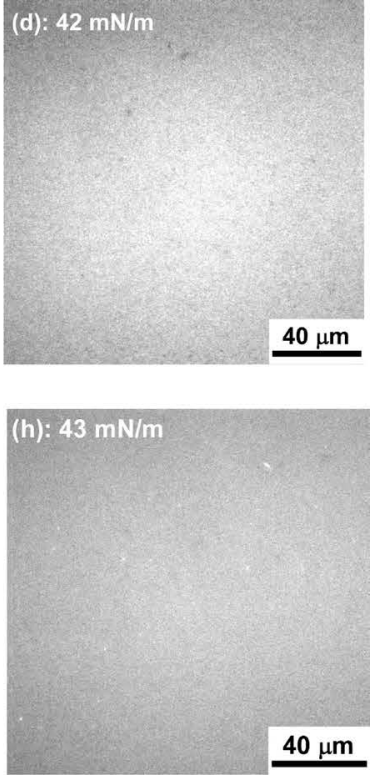

Figure 4. FM images of DPPC and DPPC-Chol mixed monolayers at various surface pressures. (a)-(d): Pure DPPC monolayer [8]; (e)-(h): Mixed monolayer of $x_{C}=0.2$.

cules would promote the condensation of the mixed monolayer (transition) at low SP. The transition at low SP is also supported by the result of STm (Figure 2) where the condensation rate has larger values than that by compression method [12] [13]. At 11 $\mathrm{mN} / \mathrm{m}$, the spot-type pattern obtained at $4 \mathrm{mN} / \mathrm{m}((\mathrm{f}))$ disappeared, and subsequently the image became homogeneously bright $((\mathrm{g}))$. Because the transition of the mixed monolayer occurred at earlier SP of $4 \mathrm{mN} / \mathrm{m}$ by Chol molecules, the condensed monolayer was formed on the water surface. A further increase in SP $(43 \mathrm{mN} / \mathrm{m})$ showed a further homogeneous image $((\mathrm{h}))$; the formation of homogeneous mixed monolayer. The brightness of image (h) was somewhat darker than that of pure DPPC monolayer $((d))$. It may be concerned to the film-thickness and refractive index between both monolayers. The mixing of Chol would thin the mean monolayer thickness of mixed monolayer compared to the pure DPPC monolayer and surface roughness also becomes larger, consequently a change in the refractive index. This interpretation would be supported from the result that the brightness of pure Chol image ((p)) was darker than (h).

On the DPPC-Chol mixed monolayer of $x_{C}=0.3$ ((i)-(l) in Figure 3), these images were quite different from those of $x_{C}=0.0$ and 0.2 . At quite a low SP of $1 \mathrm{mN} / \mathrm{m}$, the image showed line- and circle-type domain state ((i)), suggesting that aggregate of DPPC and Chol molecules was appeared on the water surface. According to an increase in SP (4 and $11 \mathrm{mN} / \mathrm{m}$ ), a number of domain appeared in the $1 \mathrm{mN} / \mathrm{m}$ increased and the domain was also enlarging gradually $((j),(k))$. The image also became bright compared to (i). At $42 \mathrm{mN} / \mathrm{m}$, the domain obtained at $11 \mathrm{mN} / \mathrm{m}$ disappeared, and subsequently the image showed homogeneous state ((l)). The brightness of image (l) was also similar to that of $x_{C}=0.2((\mathrm{~h}))$; the homogeneous mixed monolayer was formed on the water surface. These images ((i)-(l)) are similar to the monolayer formation process of 
such as stearic acid [18] and the following Chol ((m)-(p) in Figure 3), which forms the condensed monolayer on the water surface. Therefore, at $x_{C}=0.3$, a condensed monolayer was formed on the water surface from the initial stage of the monolayer formation. These are supported by the result of STm (Figure 1 and Figure 2), which the $\pi-A$ curve was similar to the Chol-like shape (not phase-transition) and the condensation rate showed maximum value at $x_{C}=0.3$.

On pure Chol monolayer, we obtained typical images that observed in the case of condensed monolayer [18]-[20]. At quite a low SP of $1 \mathrm{mN} / \mathrm{m}$, the image showed lineand circle-type domain state $((\mathrm{m}))$, as well as $x_{C}=0.3((\mathrm{I})$ in Figure 3$)$. This indicates that aggregate of Chol molecules exists on water surface. According to an increase in SP ( 3 and $8 \mathrm{mN} / \mathrm{m}$ ), a number of domain in the $1 \mathrm{mN} / \mathrm{m}$ increased and enlarged ((n), (o)). This tendency closely resembles to $x_{C}=0.3((\mathrm{j}),(\mathrm{k}))$, on the other hand, the enlargement of domain occurred at the SP lower than that $x_{C}=0.3(11 \mathrm{mN} / \mathrm{m})$. This indicates that Chol molecules themselves possess high condensation forces. At $36 \mathrm{mN} / \mathrm{m}$, the domain obtained at $8 \mathrm{mN} / \mathrm{m}$ disappeared, and subsequently the image showed homogeneous state ((p)). The brightness of image (p) was nearly the same as that of $x_{C}=0.3$ ((1)); the homogeneous Chol monolayer was formed on the water surface. This monolayer formation process of Chol ((m)-(p)) was closely similar to those of previously reported by compression method [18]-[20] and different from the case of pure DPPC monolayer, which the process by dropping method was different from that by compression method. In the case of condensable molecule itself such as Chol, the condensed monolayer would be formed on the water surface, even if using either dropping or compression method. In other words, both two methods would show the similar monolayer formation process as to the condensable molecule.

\subsection{Fluorescence Microscopy (FM)}

Figure 4 shows typical FM images on pure DPPC and DPPC-Chol mixed monolayers of $x_{C}=0.2$. Images (a)-(d) were pure DPPC monolayer $\left(x_{C}=0.0\right)$ [8]. (e)-(h) were DPPC-Chol mixed monolayer of $x_{C}=0.2$. FM observation was performed on each monolayer at four SPs of the monolayer as well as BAM, and also limited to the above two monolayers that the unique monolayer formation process using dropping method was observed by BAM.

In our previous publication, we performed FM observation of pure DPPC monolayer by dropping method and details were reported elsewhere [8]. We briefly describe the essential points. Before the SP of a transition $(\leq 8 \mathrm{mN} / \mathrm{m}$, (a) and (b)), the FM image showed gradual brightness with increase in SP, however, a few dot-type defects (black spots) were observed. Homogenous monolayer was formed on the water surface. This

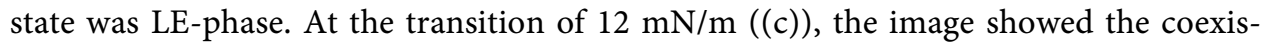
tence state that has hole-type dark parts in whole bright domain (spot-type pattern), as well as BAM ((c) in Figure 3). From the analysis of fluorescence intensity profile (not shown), the intensity of dark parts coincided with that of gray parts in (b); those parts were LE-phase and bright domain was defined semi-LC phase. As mentioned Section 
3-2, this state was contrary to that by compression method. At high SP $(42 \mathrm{mN} / \mathrm{m},(\mathrm{d}))$, the dark parts obtained in (c) disappeared, and subsequently the image became homogeneous bright. The intensity of bright parts coincided with that of bright parts in (c); homogenous semi-LC phase monolayer was formed on the water surface. The unique monolayer formation process of DPPC monolayer using dropping method was observed clearly by the FM.

On the DPPC-Chol mixed monolayer of $x_{C}=0.2((\mathrm{e})$-(h) in Figure 4), the condensation effect of Chol revealed in the FM image, clearer than BAM ((e)-(h) in Figure 3). At quite a low SP of $1 \mathrm{mN} / \mathrm{m}$, the image showed bright state ((e)) compared to that of pure DPPC monolayer of $1 \mathrm{mN} / \mathrm{m}((\mathrm{a}))$ and the irregular spot-type pattern begun to reveal partially in the image. This pattern could not be detected in the BAM image ((e) in Figure 3) because of the low resolution of BAM. Therefore, the aggregate of DPPC molecules by Chol already occurred at SP of $1 \mathrm{mN} / \mathrm{m}$. At $4 \mathrm{mN} / \mathrm{m}$, the image showed clear spot-type pattern ((f)), which an indistinct image was observed at BAM ((f) in Figure 3). The size of dark spot was smaller than that of pure DPPC monolayer at 12 $\mathrm{mN} / \mathrm{m}$. On the pure DPPC monolayer, hydrophilic monolayer is formed on the whole water surface because of easy interaction between phosphatidyl choline (hydrophilic) group of DPPC molecules and water molecules. An increase in hydrophobic interaction by the mixing of Chol weakened relatively the hydrophilic interaction, and thereby "hard-to-expand" DPPC-Chol mixture would assemble each other and form the mixed monolayer on the water surface. This corresponds to a decrease in size of dark spot, therefore, the formation of condensed monolayer, compared to the pure DPPC monolayer $((c))$. Actually, it was observed that several island-type aggregates existed in the dark spots $((\mathrm{f}))$. This state is similar to the transition state by compression method [21]. At $11 \mathrm{mN} / \mathrm{m}$, the spot-type pattern obtained at $4 \mathrm{mN} / \mathrm{m}((\mathrm{f}))$ disappeared, and the image became homogeneous bright $((\mathrm{g}))$. The intensity of bright parts coincided with that of bright parts in (f). Moreover, a further increase in SP $(43 \mathrm{mN} / \mathrm{m})$ showed a further homogeneous image $((\mathrm{h}))$ and the intensity almost coincided with that of $(\mathrm{g})$. Therefore, the homogeneous mixed monolayer was formed on the water surface, at SP more than $11 \mathrm{mN} / \mathrm{m}$.

\section{Conclusion}

In this study, we investigated the condensation effect of Chol to a DPPC monolayer formed by dropping method. The $\pi-A$ isotherm curves of various DPPC-Chol mixed monolayer showed the strong condensation effect of Chol to the fluidic DPPC monolayer and especially in the range of Chol mole fraction from $x_{C}=0.2$ to 0.4. The excess area $S_{e x}$ was about twice larger than that by compression method. The BAM and FM images showed clearly that the DPPC monolayer by dropping method has a biomembrane-like fluidic structure and is also affected strongly by the condensation effect of Chol. More than $x_{C}=0.3$, DPPC-Chol mixed monolayer showed condensed state similar to the Chol monolayer, and supported the result of $\pi-A$ isotherm curves. It is also found that the characteristics of monolayer molecules, such as those molecules are 
themselves interactively or not, comfortable with water molecules or not, sensitively respond to the difference of monolayer formation methods. A detailed understanding of these factors requires further systematic studies, using various surfactants forming the monolayer on the water surface.

\section{Acknowledgements}

This work was supported in part by a Grant-in-Aid from the Ministry of Science, Education, Sports and Culture (No. 15750118 and 21750143), DVA Medical Research and Development Funds.

\section{References}

[1] Singer, S.J. and Nicolson, G.L. (1972) The Fluid Mosaic Model of the Structure of Cell Membranes. Science, 175, 720-731. http://dx.doi.org/10.1126/science.175.4023.720

[2] Gennis, R.G. (1990) Biomembrane, Molecular Structure and Function. Springer, New York.

[3] Ohnishi, S. (1993) Seitaimaku no Doutekikouzou. 2nd Edition, University of Tokyo Press, Tokyo.

[4] Takahashi, H., Yasue, T., Ohki, K. and Hatta, I. (1996) Structure and Phase Behaviour of Dimyristoylphosphatidic Acid/poly(L-lysine) Systems. Molecular Membrane Biology, 13, 233-240. http://dx.doi.org/10.3109/09687689609160601

[5] Aloica, R.C. and Boggs, J.M. (1985) Membrane Fluidity. Academic Press, New York.

[6] Mabrey, S., Mateo, P.L. and Sturtevant, J.M. (1978) High-Sensitivity Scanning Calorimetric Study of Mixtures of Cholesterol with Dimyristoyl-and Dipalmitoylphosphatidylcholine. Biochemistry, 17, 2464-2468. http://dx.doi.org/10.1021/bi00605a034

[7] Shimabayashi, S., Terada, H. and Okabayashi, H. (1990) Seitai Colloid. Hirokawa Pub. Co., Tokyo.

[8] Yoshida, D., Yokoyama, T., Shimoaki, T., Tomita, T., Yoshida, T., Yamamoto, Y., Taga, K., Sumino, A., Dewa, T., Nango, M., Yamamoto, M. and Sheravani, Z. (2013) Morphology Observation of Dipalmitoyl Phosphatidyl Choline (DPPC) Monolayer on Water Surface by Dropping Method. Journal of Biophysical Chemistry, 4, 115-121. http://dx.doi.org/10.4236/jbpc.2013.44016

[9] Prasad, C.K., Muraleedharan, C.V. and Krishnan, L.K. (2007) Bio-Mimetic Composite Matrix That Promotes Endothelial Cell Growth for Modification of Biomaterial Surface. Journal of Biomedical Materials Research Part A, 80A, 644-654. http://dx.doi.org/10.1002/jbm.a.30968

[10] Gambinossi, F., Mecheri, B., Caminati, G., Nocentini, M., Puggelli, M. and Gabrielli, G. (2002) Antibiotic Interaction with Phospholipid Monolayers. Materials Science and Engineering: C, 22, 283-288. http://dx.doi.org/10.1016/S0928-4931(02)00187-X

[11] Kato, T., Matsumoto, N., Kawano, M., Suzuki, N. and Iriyama, K. (1994) Transition to Three-Dimensional Structures in Monolayers of a Series of Long-Chain Acids on the Water Surface by Compression Studied by Transmission Electron Microscopy and Atomic Force Microscopy. Thin Solid Films, 242, 223-228.

http://dx.doi.org/10.1016/0040-6090(94)90534-7

[12] Ohe C., Sasaki, T., Noi, M., Goto, Y. and Itoh, K. (2007) Sum Frequency Generation Spectroscopic Study of the Condensation Effect of Cholesterol on a Lipid Monolayer. Analytical and Bioanalytical Chemistry, 308, 73-39. http://dx.doi.org/10.1007/s00216-006-1030-0 
[13] Kim, K., Kim, C. and Byun, Y. (2001) Preparation of a Dipalmitoylphosphatidylcholine/ Cholesterol Langmuir-Blodgett Monolayer That Suppresses Protein Adsorption. Langmuir, 17, 5066-5070. http://dx.doi.org/10.1021/la0102096

[14] Chou, T.H. and Chang, C.H. (2000) Thermodynamic Behavior and Relaxation Processes of Mixed DPPC/Cholesterol Monolayers at the Air/Water Interface. Colloids and Surfaces B: Biointerfaces, 17, 71-79. http://dx.doi.org/10.1016/S0927-7765(99)00102-2

[15] Komada, M., Shibata, O., Nakamura, S., Lee, S. and Sugihara, G. (2004) A Monolayer Study on Three Binary Mixed Systems of Dipalmitoyl Phosphatidyl Choline with Cholesterol, Cholestanol and Stigmasterol. Colloids and Surfaces B: Biointerfaces, 33, 211-226. http://dx.doi.org/10.1016/j.colsurfb.2003.10.008

[16] Chapman, D., Owens, N.F., Phillip, M.C. and Walker, D.A. (1969) Mixed Monolayers of Phospholipids and Cholesterol. Biochimica et Biophysica Acta, 183, 458-465. http://dx.doi.org/10.1016/0005-2736(69)90160-6

[17] Worthman, L.A.D., Nag, K., Davis, P.J. and Keough, K.M. (1997) Cholesterol in Condensed and Fluid Phosphatidylcholine Monolayers Studied by Epifluorescence Microscopy. Biophysical Journal, 72, 2569-2580. http://dx.doi.org/10.1016/S0006-3495(97)78900-8

[18] Seoane, R., Miñones, J., Conde, O., Miñones Jr., J., Casas, M. and Iribarnegaray, E. (2000) Thermodynamic and Brewster Angle Microscopy Studies of Fatty Acid/Cholesterol Mixtures at the Air/Water Interface. The Journal of Physical Chemistry B, 104, 7735-7744. http://dx.doi.org/10.1021/jp001133+

[19] Flach, C.R., Mendelsohn, R., Rerek, M.E. and Moore, D.J. (2000) Biophysical Studies of Model Stratum Corneum Lipid Monolayers by Infrared Reflection-Absorption Spectroscopy and Brewster Angle Microscopy. The Journal of Physical Chemistry B, 104, 2159-2165. http://dx.doi.org/10.1021/jp9936805

[20] Mascetti, J., Castano, S., Cavagnat, D. and Desbat, B. (2008) Organization of $\beta$-Cyclodextrin under Pure Cholesterol, DMPC, or DMPG and Mixed Cholesterol/Phospholipid Monolayers. Langmuir, 24, 9616-9622. http://dx.doi.org/10.1021/la8004294

[21] Burn, A., Brezesinsuki, G., Möhwald, H., Blanzat, M., Perez, E. and Rico-Lattes, I. (2003) Interaction between Phospholipids and New Gemini Catanionic Surfactants Having Anti-HIV Activity. Colloids and Surfaces A: Physicochemical and Engineering Aspects, 228, 3-16. http://dx.doi.org/10.1016/j.colsurfa.2003.08.008

\section{Submit or recommend next manuscript to SCIRP and we will provide best service for you:}

Accepting pre-submission inquiries through Email, Facebook, LinkedIn, Twitter, etc.

A wide selection of journals (inclusive of 9 subjects, more than 200 journals)

Providing 24-hour high-quality service

User-friendly online submission system

Fair and swift peer-review system

Efficient typesetting and proofreading procedure

Display of the result of downloads and visits, as well as the number of cited articles

Maximum dissemination of your research work

Submit your manuscript at: http://papersubmission.scirp.org/

Or contact jbpc@scirp.org 